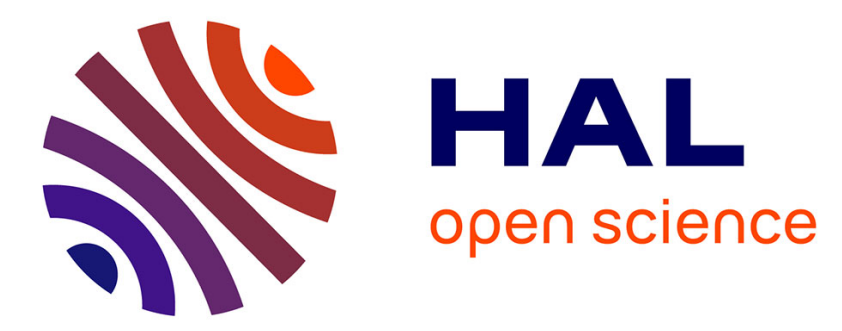

\title{
Existence and a priori estimate for elliptic problems with subquadratic gradient dependent terms
}

\author{
Nathalie Grenon, François Murat, Alessio Porreta
}

\section{To cite this version:}

Nathalie Grenon, François Murat, Alessio Porreta. Existence and a priori estimate for elliptic problems with subquadratic gradient dependent terms. 2006. hal-00112178

\author{
HAL Id: hal-00112178 \\ https://hal.science/hal-00112178 \\ Preprint submitted on 10 Nov 2006
}

HAL is a multi-disciplinary open access archive for the deposit and dissemination of scientific research documents, whether they are published or not. The documents may come from teaching and research institutions in France or abroad, or from public or private research centers.
L'archive ouverte pluridisciplinaire HAL, est destinée au dépôt et à la diffusion de documents scientifiques de niveau recherche, publiés ou non, émanant des établissements d'enseignement et de recherche français ou étrangers, des laboratoires publics ou privés. 


\title{
Existence and a priori estimate for elliptic problems with subquadratic gradient dependent terms
}

\author{
Nathalie GRENON ${ }^{a}$, François MURAT ${ }^{b}$, Alessio PORRETTA ${ }^{c}$ \\ a Centre Universitaire de Bourges, rue Gaston Berger, BP 4043, 18028 Bourges cedex, France \\ b Laboratoire Jacques-Louis Lions, Université Pierre et Marie Curie, boîte courrier 187, \\ 75252 Paris cedex 05, France \\ c Dipartimento di Matematica, Università di Roma Tor Vergata, Via della Ricerca Scientifica 1, \\ 00133 Roma, Italia
}

Abstract. In this Note we prove an a priori estimate and the existence of a solution for a class of nonlinear elliptic problems whose model is $-\operatorname{div} A(x) D u+\alpha_{0} u=\gamma|D u|^{q}+f(x)$, when $1<q<2$ and $f \in L^{m}(\Omega)$ for some suitable $m$. The main interest of the result lies in the a priori estimate, the complete proof of which is given in the Note. (C) XXXX Académie des sciences/Éditions scientifiques et médicales Elsevier SAS

Existence et estimation a priori pour des problèmes elliptiques avec des termes sous quadratiques par rappport au gradient

Résumé. Dans cette Note nous démontrons une estimation a priori et l'existence d'une solution pour une classe de problèmes non linéaires dont le modèle est -div $A(x) D u+\alpha_{0} u=$ $=\gamma|D u|^{q}+f(x)$, où $1<q<2$ et où $f \in L^{m}(\Omega)$ pour un $m$ convenable. L'intérêt principal du résultat réside dans l'estimation a priori, dont la démonstration complète est donnée dans la Note. (C) XXXX Académie des sciences/Éditions scientifiques et médicales Elsevier SAS

\section{Version française abrégée}

Dans cette Note, nous démontrons l'existence d'une solution de

$$
u \in H_{0}^{1}(\Omega), \quad-\operatorname{div} A(x) D u+a(x) u=H(x, u, D u) \quad \text { in } \mathcal{D}^{\prime}(\Omega),
$$

quand $\Omega$ est un ouvert borné de $\mathbb{R}^{N}, N \geqslant 3, A$ une matrice bornée coercive, $a$ une fonction bornée non négative (voir les hypothèses (2), (3) de la version anglaise) et quand $H: \Omega \times \mathbb{R} \times \mathbb{R}^{N} \rightarrow \mathbb{R}$ est une fonction de Carathéodory qui vérifie

$$
|H(x, s, \xi)| \leqslant \gamma|\xi|^{q}+f(x) \quad \text { avec } \gamma \geqslant 0,1+\frac{2}{N} \leqslant q<2, f \in L^{m}(\Omega) \text { et } m=\frac{N}{q^{\prime}} .
$$

Note présentée par XXX XXX

S0764-4442(00)0????-?/FLA

(c) XXXX Académie des sciences/Éditions scientifiques et médicales Elsevier SAS. Tous droits réservés. 


\section{N. Grenon et al.}

Un exemple modèle de fonction $H$ qui vérifie (4) est donné par $H(x, u, D u)=c(x) D u+d(x)|D u|^{r}+$ $+f(x)$, où $c \in L^{N}(\Omega)^{N}, 0 \leqslant r \leqslant q<2, d \in L^{s}(\Omega)$ pour un $s$ convenable et $f \in L^{p}(\Omega)$ avec $p \geqslant m=\frac{N}{q^{\prime}}$.

Le problème (1) a fait l'objet de nombreux travaux quand $H$ est à croissance quadratique ou linéaire par rapport à $D u$. Quand $q=2$, la condition (4) devient $f \in L^{\frac{N}{2}}(\Omega)$; sous cette hypothèse, l'existence d'une solution de (1) qui vérifie de plus la condition $e^{\gamma|u|}-1 \in H_{0}^{1}(\Omega)$ a été démontrée dans [10] si $\alpha_{0}=0$ et dans [8] si $\alpha_{0}>0$ (voir aussi [4], [5] dans le cas où $q=2$ et $m>\frac{N}{2}$ ). Quant à lui, le cas $q=1$ est classique, même si l'opérateur est non coercif lorsque $\gamma$ est grand; cette difficulté a été résolue dans [7]. Mais à notre connaissance, le cas $1<q<2$ est resté ouvert jusqu'à maintenant, à l'exception de l'article récent [9] (voir aussi [6]).

Dans cette Note, nous nous restreignons au cas où $q \geqslant 1+\frac{2}{N}$. En effet, dans ce cas, $m$ vérifie $m=\frac{N}{q^{\prime}} \geqslant$ $\frac{2 N}{N+2}=\left(2^{*}\right)^{\prime}$, et on a donc $f \in H^{-1}(\Omega)$, ce qui permet de chercher des solutions dans $H_{0}^{1}(\Omega)$. Dans notre futur article [11], nous traiterons aussi le cas $1<q<1+\frac{2}{N}$ par la méthode que nous utilisons ici; les résultats obtenus sont similaires même si les solutions, qui ne sont plus dans $H_{0}^{1}(\Omega)$, doivent être définies au sens des solutions renormalisées (ou d'entropie).

THÉORÈme 1. - Supposons que l'on a, outre (2), (3) et (4), l'une des deux hypothèses suivantes : ou bien $\alpha_{0}>0$, ou bien $\alpha_{0}=0$ et $\gamma^{\frac{1}{q-1}}\|f\|_{L^{m}(\Omega)}<C_{0} \alpha^{\frac{q}{q-1}}$,

où $C_{0}$ est une constante qui dépend seulement de $N$ et $q$.

Alors il existe au moins une solution u de (1) qui de plus vérifie

$$
|u|^{\sigma} \in H_{0}^{1}(\Omega) \quad \text { avec } \quad \sigma=\frac{(N-2)(q-1)}{2(2-q)} .
$$

De plus, toute solution de (1) qui vérifie la condition de régularité (7) vérifie l'estimation a priori

$$
\|u\|_{H_{0}^{1}(\Omega)}+\left\||u|^{\sigma}\right\|_{H_{0}^{1}(\Omega)} \leqslant M,
$$

où $M$ depend seulement de $N, q,|\Omega|, \alpha, \alpha_{0}, \gamma$ et $f$.

Quand $\alpha_{0}>0$, la constante $M$ qui apparaît dans l'estimation a priori (8) dépend de la fonction $f$, non seulement par l'intermédiaire de sa norme $\|f\|_{L^{m}(\Omega)}$, mais aussi par l'intermédiaire du nombre $k^{*}$ défini par (17). Cependant la constante $M$ est bornée quand $f$ varie dans un ensemble de fonctions qui sont bornées et équi-intégrables dans $L^{m}(\Omega)$.

La relation entre les paramètres $q$ et $m$ imposée dans (4) est naturelle, car les conditions nécessaires pour l'existence d'une solution de (1) démontrées dans [1], [12] conduisent dans le cadre adopté ici à $m=\frac{N}{q^{\prime}}$. De plus ces résultats montrent qu'une condition sur la taille des données est nécessaire pour l'existence d'une solution de (1) quand $\alpha_{0}=0$.

L'exposant $\sigma$ défini par (7) vérifie $\sigma \geqslant 1$ quand $q \geqslant 1+\frac{2}{N}$.

Il existe des solutions de (1) qui ne vérifient pas la condition de régularité (7). Un exemple classique en est $u(x)=C_{q}\left(|x|^{-\frac{2-q}{q-1}}-1\right)$, qui, lorsque $q>1+\frac{2}{N}$, vérifie, pour un choix convenable de $C_{q}, u \in H_{0}^{1}(\Omega)$, $-\Delta u=|D u|^{q}$ in $\mathcal{D}^{\prime}\left(B_{1}\right)$ et $|u|^{\rho} \in H_{0}^{1}(\Omega)$ pour tout $\rho<\sigma$, mais ne vérifie pas $|u|^{\sigma} \in H_{0}^{1}(\Omega)$. Par contre, des résultats d'unicité pour les solutions de (1) qui vérifient (7) ont été récemment démontrés dans [2].

Dans notre futur article [11], nous donnerons un certain nombre de généralisations du Théorème 1. Comme nous l'avons déja dit, nous traiterons tout l'intervalle $1<q<2$. Nous étudierons aussi le cas des conditions aux limites de Neumann et de Fourier, le cas des ouverts non bornés, et celui de fonctions $f$ appartenant à des espaces de Lorentz ou à des espaces de Sobolev négatifs. Tout cela sera présenté dans le cadre d'opérateurs non linéaires pseudomonotones de type Leray-Lions définis dans l'espace $W^{1, p}(\Omega)$. Nous considérerons plus tard l'analogue parabolique du problème (1). 


\section{Introduction, main result and comments}

In this Note we prove the existence of a solution of

$$
u \in H_{0}^{1}(\Omega), \quad-\operatorname{div} A(x) D u+a(x) u=H(x, u, D u) \quad \text { in } \mathcal{D}^{\prime}(\Omega),
$$

when $\Omega$ is an open bounded set of $\mathbb{R}^{N}(N \geqslant 3), A$ a bounded coercive matrix, $a$ a bounded nonnegative function, i.e.,

$$
\begin{gathered}
A \in L^{\infty}(\Omega)^{N \times N}, \quad A \geqslant \alpha I, \quad \alpha>0, \\
a \in L^{\infty}(\Omega), \quad a \geqslant \alpha_{0}, \quad \alpha_{0} \geqslant 0,
\end{gathered}
$$

and when $H: \Omega \times \mathbb{R} \times \mathbb{R}^{N} \rightarrow \mathbb{R}$ is a Carathéodory function which satisfies

$$
|H(x, s, \xi)| \leqslant \gamma|\xi|^{q}+f(x) \quad \text { with } \gamma \geqslant 0,1+\frac{2}{N} \leqslant q<2, f \in L^{m}(\Omega) \text { and } m=\frac{N}{q^{\prime}} .
$$

A model example of function $H$ which satisfies (4) is $H(x, u, D u)=c(x) D u+d(x)|D u|^{r}+f(x)$ with $c \in L^{N}(\Omega)^{N}, 0 \leqslant r \leqslant q<2, d \in L^{s}(\Omega)$ for a suitable $s$ and $f \in L^{p}(\Omega)$ with $p \geqslant m=\frac{N}{q^{\prime}}$.

There is a wide literature concerning problem (1) when $H$ has a quadratic or a linear growth with respect to $D u$. When $q=2$, condition (4) becomes $f \in L^{\frac{N}{2}}(\Omega)$; in this case existence of a solution of (1) which satisfies the further regularity $e^{\gamma|u|}-1 \in H_{0}^{1}(\Omega)$ has been proved in [10] if $\alpha_{0}=0$ and in [8] if $\alpha_{0}>0$ (previous references for the case where $q=2$ and $m>\frac{N}{2}$ are, e.g., [4], [5]). On the other hand, the case $q=1$ is classical, but exhibits an important difficulty when $\gamma$ is large, due to the fact that the operator is then non coercive; this problem has been solved in [7]. As far as we know, the case $1<q<2$ has been left open until now, except for the very recent paper [9] (see also [6]).

In the present Note, we restrict ourselves to the case where $q \geqslant 1+\frac{2}{N}$. Indeed in this case $m$ satisfies $m=\frac{N}{q^{\prime}} \geqslant \frac{2 N}{N+2}=\left(2^{*}\right)^{\prime}$, hence $f \in H^{-1}(\Omega)$. This allows us to look for solutions which belong to $H_{0}^{1}(\Omega)$. In our forthcoming paper [11] we will also treat the case $1<q<1+\frac{2}{N}$ by the method used in the present Note and obtain very similar results, except for the fact that the solution is no more in $H_{0}^{1}(\Omega)$ and has to be defined as a renormalized (or entropy) solution.

Theorem 1. - Assume (2), (3), (4), and one of the two following hypotheses:

$$
\begin{gathered}
\text { either } \alpha_{0}>0, \\
\text { or } \alpha_{0}=0 \quad \text { and } \gamma^{\frac{1}{q-1}}\|f\|_{L^{m}(\Omega)}<C_{0} \alpha^{\frac{q}{q-1}},
\end{gathered}
$$

where $C_{0}$ is a constant which depends only on $N$ and $q$.

Then there exists at least one solution $u$ of (1) which further satisfies

$$
|u|^{\sigma} \in H_{0}^{1}(\Omega) \text { with } \sigma=\frac{(N-2)(q-1)}{2(2-q)} .
$$

Moreover, every solution of (1) which satisfies the regularity requirement (7) satisfies the estimate

$$
\|u\|_{H_{0}^{1}(\Omega)}+\left\||u|^{\sigma}\right\|_{H_{0}^{1}(\Omega)} \leqslant M,
$$

where $M$ depends only on $N, q,|\Omega|, \alpha, \alpha_{0}, \gamma$ and $f$.

Remark 2. - When $\alpha_{0}>0$, the constant $M$ which appears in the a priori estimate (8) depends on the function $f$, not only through its norm $\|f\|_{L^{m}(\Omega)}$, but also through the number $k^{*}$ defined by (17) below. However, the constant $M$ is bounded when $f$ varies in a set of functions which are bounded and equiintegrable in $L^{m}(\Omega)$. 


\section{N. Grenon et al.}

Remark 3. - The link imposed in (4) between the parameters $q$ and $m$ is natural. Consider indeed the model problem $u \in H_{0}^{1}(\Omega),-\Delta u+u=\gamma|D u|^{q}+f(x)$ in $\mathcal{D}^{\prime}(\Omega)$, with $1<q<2$ and $f \in L^{m}(\Omega)$ for some $m$. Since $f \in L^{m}(\Omega)$, one expects, in view of the $W^{2, p}$ regularity result, that $u \in W^{2, m}(\Omega)$, which in turns implies, by reading the equation, that $|D u|^{q} \in L^{m}(\Omega)$. But $W^{2, m}(\Omega) \subset W^{1, m^{*}}(\Omega)$ implies $|D u| \in L^{m^{*}}(\Omega)$. This leads to $q m=m^{*}$, namely $m=\frac{N}{q^{\prime}}$ as required. On the other hand, the necessary conditions obtained in [1], [12] for the existence of a solution of (1), when specialized to the present setting, lead to the condition $m=\frac{N}{q^{\prime}}$. Moreover, these papers show that a condition like (6) on the size of the data is necessary in order to have the existence of a solution of (1) when $\alpha_{0}=0$.

Remark 4. - The exponent $\sigma$ defined by (7) satisfies $\sigma \geqslant 1$ when $q \geqslant 1+\frac{2}{N}$.

Remark 5. - There exist solutions of (1) which do not satisfy the regularity (7). A well-known example is the function $u(x)=C_{q}\left(|x|^{-\frac{2-q}{q-1}}-1\right)$, which, when $q>1+\frac{2}{N}$, satisfies, for a suitable choice of $C_{q}, u \in H_{0}^{1}(\Omega),-\Delta u=|D u|^{q}$ in $\mathcal{D}^{\prime}\left(B_{1}\right)$ and $|u|^{\rho} \in H_{0}^{1}(\Omega)$ for every $\rho<\sigma$, but does not satisfy $|u|^{\sigma} \in H_{0}^{1}(\Omega)$. In contrast, uniqueness results for the solutions of (1) which satisfy the regularity requirement (7) have been recently proved in [2].

Remark 6. - In our forthcoming paper [11], we will present many extensions of Theorem 1. As said before, we will consider, as far as the growth of $H(x, u, D u)$ with respect to $|D u|$ is concerned, the full range $1<q<2$. We will also treat the case of Neumann's and Robin's boundary conditions, the case of unbounded domains and the case of functions $f$ in some Lorentz spaces and in some negative Sobolev spaces. All of this will be done for general nonlinear pseudomonotone operators of Leray-Lions type defined in $W^{1, p}(\Omega)$. We will consider the parabolic analogue of problem (1) later.

\section{Proof of Theorem 1}

In this proof, $C_{0}, C_{1}, C_{2}, C_{3}$ and the generic constant $C$ will denote different positive constants which depend only on $N$ and $q$.

We first prove the second part of Theorem 1, namely the a priori estimate (8).

Let $u$ be any solution of (1) which satisfies the regularity requirement (7). In equation (1) we can take as test function any function $v \in H_{0}^{1}(\Omega) \cap L^{\infty}(\Omega)$, but also $v=|u|^{2 \sigma-2} u$ (recall that $\sigma \geqslant 1$ ): to prove this assertion, take $v=\left|T_{n}(u)\right|^{2 \sigma-2} T_{n}(u)$, where $T_{n}$ is the truncation at height $n$, and pass to the limit using (4) and (7). We actually use a slight modification of the latest test function: given $k>0$, we choose as test function in (1) $v=\left|G_{k}(u)\right|^{2 \sigma-2} G_{k}(u)$, where $G_{k}(s)=s-T_{k}(s)$. We obtain

$$
\begin{aligned}
& \alpha(2 \sigma-1) \int_{\Omega}\left|G_{k}(u)\right|^{2 \sigma-2}\left|D G_{k}(u)\right|^{2} d x+\alpha_{0} \int_{\Omega}|u|\left|G_{k}(u)\right|^{2 \sigma-1} d x \leqslant \\
& \quad \leqslant \gamma \int_{\Omega}\left|D G_{k}(u)\right|^{q}\left|G_{k}(u)\right|^{2 \sigma-1} d x+\int_{\Omega}|f|\left|G_{k}(u)\right|^{2 \sigma-1} d x .
\end{aligned}
$$

The first term of the left-hand side of (9) is nothing but

$$
\alpha(2 \sigma-1) \int_{\Omega}\left|G_{k}(u)\right|^{2 \sigma-2}\left|D G_{k}(u)\right|^{2} d x=\alpha C \int_{\Omega}\left|D\left(\left|G_{k}(u)\right|^{\sigma}\right)\right|^{2} d x .
$$

We then estimate the first term of the right-hand side of (9); Hölder's inequality and the same computation as in (10) yield

$$
\begin{aligned}
\gamma \int_{\Omega}\left|D G_{k}(u)\right|^{q}\left|G_{k}(u)\right|^{2 \sigma-1} d x & =\gamma \int_{\Omega}\left|D G_{k}(u)\right|^{q}\left|G_{k}(u)\right|^{\frac{2 \sigma-2}{2} q}\left|G_{k}(u)\right|^{2 \sigma-1-(\sigma-1) q} d x \leqslant \\
& \leqslant \gamma C\left(\int_{\Omega}\left|D\left(\left|G_{k}(u)\right|^{\sigma}\right)\right|^{2} d x\right)^{\frac{q}{2}}\left(\int_{\Omega}\left|G_{k}(u)\right|^{(2 \sigma-1-(\sigma-1) q) \frac{2}{2-q}} d x\right)^{1-\frac{q}{2}}
\end{aligned}
$$


But $(2 \sigma-1-(\sigma-1) q) \frac{2}{2-q}=\sigma 2^{*}$, and Sobolev's embedding yields

$$
\gamma \int_{\Omega}\left|D G_{k}(u)\right|^{q}\left|G_{k}(u)\right|^{2 \sigma-1} d x \leqslant \gamma C\left\|D\left(\left|G_{k}(u)\right|^{\sigma}\right)\right\|_{L^{2}(\Omega)}^{q+\left(1-\frac{q}{2}\right) 2^{*}}
$$

We finally estimate the second term of the right-hand side of (9) by (note that $|u|>k$ when $G_{k}(u) \neq 0$ )

$$
\begin{aligned}
\int_{\Omega}|f|\left|G_{k}(u)\right|^{2 \sigma-1} d x & =\int_{\left\{|f| \leqslant \alpha_{0}|u|\right\}}|f|\left|G_{k}(u)\right|^{2 \sigma-1} d x+\int_{\left\{|f|>\alpha_{0}|u|\right\}}|f|\left|G_{k}(u)\right|^{2 \sigma-1} d x \leqslant \\
& \leqslant \alpha_{0} \int_{\Omega}|u|\left|G_{k}(u)\right|^{2 \sigma-1} d x+\int_{\left\{|f|>\alpha_{0} k\right\}}|f|\left|G_{k}(u)\right|^{2 \sigma-1} d x .
\end{aligned}
$$

The first term of the right-hand side of (12) is absorbed by the second term of the left-hand side of (9). Using Hölder's inequality, and since $(2 \sigma-1) m^{\prime}=\sigma 2^{*}$, we estimate the second term by

$$
\begin{aligned}
\int_{\left\{|f|>\alpha_{0} k\right\}}|f|\left|G_{k}(u)\right|^{2 \sigma-1} d x & \leqslant\left\|f \chi_{\left\{|f|>\alpha_{0} k\right\}}\right\|_{L^{m}(\Omega)}\left\|\left|G_{k}(u)\right|^{2 \sigma-1}\right\|_{L^{m^{\prime}}(\Omega)} \leqslant \\
& \leqslant C\left\|f \chi_{\left\{|f|>\alpha_{0} k\right\}}\right\|_{L^{m}(\Omega)}\left\|D\left(\left|G_{k}(u)\right|^{\sigma}\right)\right\|_{L^{2}(\Omega)}^{\frac{2}{m}^{*}} .
\end{aligned}
$$

Set

$$
Y_{k}=\left\|D\left(\left|G_{k}(u)\right|^{\sigma}\right)\right\|_{L^{2}(\Omega)} .
$$

Using (10), (11), (12) and (13) in (9) yields, for two positive constants $C_{1}$ and $C_{2}$

$$
\alpha C_{1} Y_{k}^{2} \leqslant \gamma C_{2} Y_{k}^{q+\left(1-\frac{1}{q}\right) 2^{*}}+\left\|f \chi_{\left\{|f|>\alpha_{0} k\right\}}\right\|_{L^{m}(\Omega)} Y_{k}^{\frac{2^{*}}{m^{\prime}}}
$$

Dividing by $Y_{k}^{\frac{2^{*}}{m^{\prime}}}$, and using $2-\frac{2^{*}}{m^{\prime}}=\frac{2^{*}}{N}\left(q^{\prime}-2\right)$ and $q+\left(1-\frac{q}{2}\right) 2^{*}-\frac{2^{*}}{m^{\prime}}=\frac{2^{*}}{N}\left(q^{\prime}-q\right)$, we finally obtain

$$
\forall k \geqslant 0, \quad \alpha C_{1} Y_{k}^{\frac{2^{*}}{N}\left(q^{\prime}-2\right)}-\gamma C_{2} Y_{k}^{\frac{2^{*}}{N}\left(q^{\prime}-q\right)} \leqslant\left\|f \chi_{\left\{|f|>\alpha_{0} k\right\}}\right\|_{L^{m}(\Omega)}
$$

Define the function $F: \mathbb{R}^{+} \rightarrow \mathbb{R}$ by

$$
F(Y)=\alpha C_{1} Y^{2^{*}}\left(q^{\prime}-2\right)-\gamma C_{2} Y^{\frac{2^{*}}{N}\left(q^{\prime}-q\right)}
$$

Then (14) is equivalent to

$$
\forall k \geqslant 0, \quad F\left(Y_{k}\right) \leqslant\left\|f \chi_{\left\{|f|>\alpha_{0} k\right\}}\right\|_{L^{m}(\Omega)} .
$$

Since $q<2, F$ is a concave function with a unique maximizer $Z^{*}$ and maximum $F^{*}$, where $Z^{*}$ and $F^{*}$ are given by

$$
Z^{*}=C_{3}\left(\frac{\alpha}{\gamma}\right)^{\frac{N}{2^{*}(2-q)}} \quad \text { and } \quad F^{*}=F\left(Z^{*}\right)=C_{0} \frac{\alpha^{\frac{q}{q-1}}}{\gamma^{\frac{1}{q-1}}} .
$$

Inequality (15) is non trivial only if its right-hand side is strictly smaller than $F^{*}$.

Here we split the proof into two cases.

(i) If $\alpha_{0}=0$, hypothesis (6) is nothing but $\|f\|_{L^{m}(\Omega)}<F^{*}$. Then equation $F(Y)=\|f\|_{L^{m}(\Omega)}$ has two roots $Z_{0}^{-}$and $Z_{0}^{+}$, with $0<Z_{0}^{-}<Z^{*}<Z_{0}^{+}$, and inequality (15) is equivalent to

$$
\forall k \geqslant 0, \quad \text { either } \quad Y_{k} \leqslant Z_{0}^{-} \quad \text { or } \quad Y_{k} \geqslant Z_{0}^{+} \text {. }
$$




\section{N. Grenon et al.}

But since $|u|^{\sigma} \in H_{0}^{1}(\Omega)$, the function $k \rightarrow Y_{k}=\left\|D\left(\left|G_{k}(u)\right|^{\sigma}\right)\right\|_{L^{2}(\Omega)}$ is continuous and tends to zero when $k$ tends to infinity. The alternative (16) then implies that $Y_{k} \leqslant Z_{0}^{-}$for every $k$; in particular, one has

$$
Y_{0}=\left\|D\left(|u|^{\sigma}\right)\right\|_{L^{2}(\Omega)} \leqslant Z_{0}^{-}<Z^{*}
$$

(ii) If $\alpha_{0}>0$, we define $k^{*}$ as

$$
k^{*}=\inf \left\{k>0:\left\|f \chi_{\left\{|f|>\alpha_{0} k\right\}}\right\|_{L^{m}(\Omega)}<F^{*}\right\} .
$$

For every $\delta>0$, one has $\left\|f \chi_{\left\{|f|>\alpha_{0}\left(k^{*}+\delta\right)\right\}}\right\|_{L^{m}(\Omega)}<F^{*}$, and equation $F(Y)=\left\|f \chi_{\left\{|f|>\alpha_{0}\left(k^{*}+\delta\right)\right\}}\right\|_{L^{m}(\Omega)}$ has two roots $Z_{k^{*}+\delta}^{-}$and $Z_{k^{*}+\delta}^{+}$, with $0<Z_{k^{*}+\delta}^{-}<Z^{*}<Z_{k^{*}+\delta}^{+}$. Inequality (15) implies that for every $k \geqslant k^{*}+\delta$, either $Y_{k} \leqslant Z_{k^{*}+\delta}^{-}$, or $Y_{k} \geqslant Z_{k^{*}+\delta}^{+}$. But the function $k \rightarrow Y_{k}$ is continuous and tends to zero when $k$ tends to infinity. We conclude that for every $k \geqslant k^{*}+\delta$, one has $Y_{k} \leqslant Z_{k^{*}+\delta}^{-}$, and in particular that $Y_{k^{*}+\delta}=\left\|D\left(\left|G_{k^{*}+\delta}(u)\right|^{\sigma}\right)\right\|_{L^{2}(\Omega)} \leqslant Z_{k^{*}+\delta}^{-}<Z^{*}$. We then let $\delta$ tend to zero.

In both cases, we have proved that

$$
\left\|D\left(\left|G_{k^{*}}(u)\right|^{\sigma}\right)\right\|_{L^{2}(\Omega)} \leqslant Z^{*}=C_{3}\left(\frac{\alpha}{\gamma}\right)^{\frac{N}{2^{*}(2-q)}}
$$

where $k^{*}=0$ when $\alpha_{0}=0$, and where $k^{*}$ is defined by (17) when $\alpha_{0}>0$. When $k^{*}=0$, inequality (18) is nothing but the second part of the a priori estimate (8). Note that the constant $Z^{*}$, which plays here a role similar to the constant $M$, depends only on $N, q, \alpha$ and $\gamma$, but that $k^{*}$ depends on the function $f$ itself.

We now prove the first part of the a priori estimate (8). Since one has $D G_{k_{1}^{*}}(u)=\chi_{\left\{x:|u| \geqslant k_{1}^{*}\right\}} D u$ and $\left|D\left(\left|G_{k^{*}}(u)\right|^{\sigma}\right)\right|=\sigma\left|G_{k^{*}}(u)\right|^{\sigma-1}\left|D G_{k^{*}}(u)\right|$, estimate (18) provides an estimate of $\left\|D G_{k_{1}^{*}}(u)\right\|_{L^{2}(\Omega)}$ for $k_{1}^{*}=k^{*}+1$. We then use $v=T_{k_{1}^{*}}(u)$ in (1) and we get

$$
\begin{aligned}
\alpha \int_{\Omega}\left|D T_{k_{1}^{*}}(u)\right|^{2} d x & \leqslant \int_{\Omega}\left(\gamma|D u|^{q}+f\right)\left|T_{k_{1}^{*}}(u)\right| d x \leqslant \leqslant \gamma k_{1}^{*} \int_{\Omega}|D u|^{q} d x+k_{1}^{*}|| f \|_{L^{1}(\Omega)} \leqslant \\
& \leqslant \gamma k_{1}^{*} \int_{\Omega}\left|D T_{k_{1}^{*}}(u)\right|^{q} d x+\gamma k_{1}^{*} \int_{\Omega}\left|D G_{k_{1}^{*}}(u)\right|^{q} d x+k_{1}^{*}|| f \|_{L^{1}(\Omega)},
\end{aligned}
$$

from which we deduce, using $q<2$ and the estimate on $\left\|D G_{k_{1}^{*}}(u)\right\|_{L^{2}(\Omega)}$, an estimate on $\left\|D T_{k_{1}^{*}}(u)\right\|_{L^{2}(\Omega)}$, and thus the first part of the a priori estimate (8), with a constant which depends on $k^{*}$.

Finally combining the estimates on $\left\|D T_{k_{1}^{*}}(u)\right\|_{L^{2}(\Omega)}$ and $\left\|D G_{k_{1}^{*}}(u)\right\|_{L^{2}(\Omega)}$ and estimate (18) completes the proof of the second part of the a priori estimate (8). The constant which appears in this estimate depends on $k^{*}$.

We now pass to the proof of the existence of a solution of (1) which satisfies the regularity requirement (7). This proof is classical. One considers the approximation of (1) by the problem in which the function $H$ is replaced by the function $H_{\varepsilon}=T_{\frac{1}{\varepsilon}}(H)$; this function $H_{\varepsilon}$ satisfies (4) for every $\varepsilon>0$. This equation has at least one solution $u_{\varepsilon}$ which, by the weak maximum principle, belongs to $H_{0}^{1}(\Omega) \cap L^{\infty}(\Omega)$. Therefore $u_{\varepsilon}$ satisfies the regularity requirement (7), and the a priori estimate (8) ensures that $u_{\varepsilon}$ and $\left|u_{\varepsilon}\right|^{\sigma}$ are bounded in $H_{0}^{1}(\Omega)$. In view of the growth condition (4), $H_{\varepsilon}\left(x, u_{\varepsilon}, D u_{\varepsilon}\right)$ is then bounded in $L^{\frac{2}{q}}(\Omega)$. Let $u \in H_{0}^{1}(\Omega)$ be such that a subsequence (still denoted by $\varepsilon$ ) $u_{\varepsilon}$ weakly converges to $u$ in $H_{0}^{1}(\Omega)$. The bound of $u_{\varepsilon}$ in $H_{0}^{1}(\Omega)$ implies that $-\operatorname{div} A(x) D u_{\varepsilon}$ is bounded both in $H^{-1}(\Omega)$ and in $L^{\frac{2}{q}}(\Omega)$. These bounds imply (see, e.g., [3]) that, extracting if necessary a new subsequence, $D u_{\varepsilon}$ converges to $D u$ almost everywhere in $\Omega$, which in turn implies that $H_{\varepsilon}\left(x, u_{\varepsilon}, D u_{\varepsilon}\right)$ converges to $H(x, u, D u)$ strongly in $L^{s}(\Omega)$ for every $s<\frac{2}{q}$. This result easily allows one to pass to the limit in the approximate equation, which proves the existence of a solution of (1) which satisfies (7). 


\section{References}

[1] N. Alaa and M. Pierre, Weak solutions of some quasilinear elliptic equations with data measures, SIAM J. Math. Anal. 24 (1993), 23-35.

[2] G. Barles and A. Porretta, Uniqueness for unbounded solutions to stationary viscous Hamilton-Jacobi equations, preprint.

[3] L. Boccardo and F. Murat, Almost everywhere convergence of the gradients of solutions to elliptic and parabolic equations, Nonlinear Anal. TMA 19 (1992), 581-597.

[4] L. Boccardo, F. Murat and J.-P. Puel, Existence de solutions faibles pour des équations elliptiques quasi-linéaires à croissance quadratique, in Nonlinear partial differential equations and their applications, Collège de France Seminar, Vol. IV, ed. H. Brezis and J.-L. Lions, Pitman Research Notes in Mathematics 84, London, (1983), 19-73.

[5] L. Boccardo, F. Murat and J.-P. Puel, $L^{\infty}$ Estimates for some nonlinear partial differential equations and an application to an existence result, SIAM J. Math. Anal. 23 (1992), 326-333.

[6] L. Boccardo and M.M. Porzio, Quasilinear elliptic equations with subquadratic growth, preprint.

[7] G. Bottaro and M.E. Marina, Problema di Dirichlet per equazioni ellittiche di tipo variazionale su insiemi non limitati, Boll. Un. Mat. Ital. 8 (1973), 46-56.

[8] A. Dall'Aglio, D. Giachetti and J.-P. Puel, Nonlinear elliptic equations with natural growth in general domains, Ann. Mat. Pura Appl. 181 (2002), 407-426.

[9] V. Ferone and B. Messano, Comparison results for nonlinear equations with general growth in the gradient, preprint.

[10] V. Ferone and F. Murat, Quasilinear problems having quadratic growth in the gradient: an existence result when the source term is small, in Equations aux dérivées partielles et applications, Articles dédiés à Jacques-Louis Lions, Gauthier-Villars, Paris, (1998), 497-515.

[11] N. Grenon, F. Murat and A. Porretta, Elliptic equations with superlinear gradient dependent terms: existence and a priori estimates, in preparation.

[12] K. Hansson, V. Maz'ya and I. Verbitsky, Criteria of solvability for multidimensional Riccati's equation, Arkiv för Mat. 37 (1999), 87-120. 